Acta Theriologica, Suppl. 4: 57-66, 1997.

PL ISSN 0001-7051

\title{
Phenotypic diversity and population dynamics: another look (with particular reference to the common shrew Sorex araneus)
}

\author{
Vladimir M. ZAKHAROV, Erkki PANKAKOSKI and Boris I. SHEFTEL
}

\begin{abstract}
Zakharov V. M., Pankakoski E. and Sheftel B. I. 1997. Phenotypic diversity and population dynamics: another look (with particular reference to the common shrew Sorex araneus). [In: Developmental homeostasis in natural populations of mammals: phenetic approach. V. M. Zakharov and A. V. Yablokov, eds]. Acta Theriologica, Suppl. 4: $57-66$.
\end{abstract}

We studied temporal changes in the level of phenotypic diversity, measured by the total phenotypic variance for several characters of skull morphology, in two populations of the common shrew Sorex araneus Linnaeus, 1758 . We compared a population from central Siberia having a high-amplitude four-year cycle with a population from southern Finland having noncyclic dynamics. The level of total phenotypic diversity varied significantly among years in both populations, but was correlated neither with density nor with breeding success in either of them. We did, however, find differences between the two populations. When we compared changes in the level of phenotypic diversity with changes in the level of developmental stability, as measured by chance developmental variance (fluctuating asymmetry), the cyclic Siberian population exhibited increased developmental variability in the peak year, which was associated with relatively small proportion of other sources of variation (and genetic variation in particular). In other years, the role of chance variation was less and the proportion of other sources of variance was higher. On the other hand, in the noncyclic Finnish population, oscillations in the level of phenotypic diversity were mainly caused by changes in developmental stability. These results illustrate that not only dynamics of genotype variety, but also the alterations in the level of developmental stability can be of great importance for changes in phenotypic diversity.

N. K. Koltzov Institute of Developmental Biology Russian Academy of Sciences, Vavilov Str. 26, Moscow, 117808 Russia (VMZ); Department of Ecology and Systematics, Division of Population Biology, P. O. Box 17 (Arkadiankatu 7), FIN-00014, University of Helsinki, Finland (EP); Institute of Evolutionary and Ecological Problems Russian Academy of Science, Leninsky Prospekt 33, Moscow, 117071 Russia (BIS)

Key words: Sorex araneus, fluctuating asymmetry, phenotypic diversity, developmental stability, population dynamics

\section{Introduction}

Temporal changes in the level of phenotypic diversity are important for understanding population processes, as well as for revealing differences in phenotypic diversity among populations (Yablokov 1986, Zakharov 1987, 1989). From a genetic point of view, where phenotypic diversity is assumed to reflect genetic 
variability, we would predict that an increase in the number of individuals in a population would lead to an increase in variability, if it could be allowed by natural selection. But such an interpretation assumes that phenotypic variation has a genetic basis. The apportioning of variation into genetic or environmental causes has been a complicated task for a majority of population studies. For this we have to move from estimating phenotypic diversity as a phenomenon to revealing the causes of variability (Langlet 1971, Philiptschenko 1978, Zakharov 1992). It could be possible by comparing the total phenotypic variance with the variance reflecting chance developmental variability (Thoday 1953, Reeve 1960, Bader 1965, Zakharov 1987, 1989).

The total phenotypic variance consists of three main types of variance: genetic variance (caused by genotypic diversity), environmental variance (reflecting the variety of environmental conditions), and chance developmental variance (estimating the level of ontogenetic accidents attributable to neither genetic nor environmental variation) (Astauroff 1930, Mather 1953, Thoday 1958, Rasmuson 1960, Reeve 1960, Soulé 1982, Pankakoski et al. 1987, Zakharov 1992). Chance developmental variance is an important component of the total phenotypic variance because it reflects the level of developmental stability (the ability to develop the same phenotype under particular environmental conditions). Consequently, it is a general ontogenetic characteristic that is very sensitive to various environmental stresses (Valentine et al. 1973, Zakharov 1987, 1989, Leary and Allendorf 1989, Leary et al. 1992, Pankakoski et al. 1992, Siegel et al. 1992).

The easiest way to estimate the chance developmental variance is to measure the difference between homologous structures on the right and left sides of the same organism, where on average these structures are symmetric. The direct comparison of the total phenotypic variance and the chance developmental variance allows one to estimate the proportion of variation caused by developmental disturbances and a role of the others and especially that of the genetic one (Zakharov 1987), because the variety of environments in the certain area under study inhabited by the same population commonly proves to be rather stable in time.

In this study we try to reveal possible temporal changes in the level of phenotypic diversity measured by the total phenotypic variance of skull characters in two populations of common shrew Sorex araneus Linnaeus, 1758 characterized by different types of dynamics. One population, from central Siberia, has a high-amplitude four-year cycle and another, from southern Finland, is noncyclic (Pankakoski 1985, Sheftel 1989).

We also compare the observed temporal pattern in total phenotypic diversity with that of developmental stability described earlier for the same set of morphological characters in the same population. In the earlier study (Zakharov et al. 1991) we found that the level of developmental stability was negatively correlated with density in a cyclic population, but not in a noncyclic population. Comparison of the observed patterns of variation in total phenotypic diversity with those for 
developmental stability could provide us with information about what fraction of the temporal pattern of variation in total phenotypic diversity is due to variation in developmental stability, and whether this fraction differs between the two populations.

\section{Material and methods}

Common shrews Sorex araneus were collected from two populations: central Siberia (1977-1982) and southern Finland (1975-1980). Details for trapping techniques, localities as well as for calculating the estimates of density and breeding success have been described earlier (Pankakoski 1985, Sheftel 1989, Zakharov et al. 1991). The number of individuals studied was 254 for Siberia and 170 for southern Finland.

Previous studies have revealed a highly regular four-year population cycle in central Siberia and relatively stable shrews density in southern Finland (Fig. 1). In the Siberian population, density is negatively correlated with breeding success, but these two parameters are not as closely correlated in the Finnish population. The data suggest, that in the Siberian population, overpopulation may adversely affect the condition of individuals, while in the Finnish population changes in environmental factors could be of primary importance (Zakharov et al. 1991).

To measure the level of phenotypic diversity, quantitative skull foramen characters (13 for the Siberian population and 30 for the Finnish population) were analyzed. Foramina are small openings through the bone in the skull for nerves, blood vessels, and tooth roots (Pankakoski and Hanski 1989). Foramen counts were the same as those used in our earlier study (Zakharov et al. 1991).

As all characters under study were bilateral, the sum of the number of foramina on the left (l) and right $(r)$ sides of the skull $(1+r)$ has been considered as the main variable. The total phenotype variance $[\operatorname{Var}(\mathrm{l}+\mathrm{r})]$ has been used to measure the level of phenotypic diversity.

Another variance, that of $(1-r)$, was calculated using the same data. Such a variance for the difference between two sides of the body reflects the level of fluctuating asymmetry, or small non-directional deviations from symmetry (Van Valen 1962, Soulé 1967, Palmer and Strobeck 1986, Zakharov 1987, Leary and Allendorf 1989). This variance of $(1-r)$ is a good measure of developmental stability (Mather 1953, Thoday 1958, Zakharov 1981, 1989). When applied to populations of shrews, we showed (Zakharov et al. 1991) that chance developmental variability, variance (l-r), mainly reflects the average condition of individuals within populations. Its annual values were positively correlated with annual estimates of density only in the cyclical Siberian population, while, as a non-specific measure of stress, it showed negative interaction with breeding success in both populations (Fig. 1).

Thus, we have calculated variance $(l+r)$, total phenotypic diversity, and variance $(l-r)$, chance developmental variability, of the values for the same characters on two sides of the body. As a result, we can compare these statistical indices for the same data.

Statistically, the value of variance for sum is equal to the value of variance for difference when correlation between 1 and $\mathrm{r}$ is zero (Sokal and Rohlf 1981). Correspondingly, if the correlation increases, the variance for the sum becomes higher than the variance for the difference.

Biologically, Astauroff (1930) established that if there is only one source of variation in a group of individuals, chance disturbances during individual development, then the total phenotypic diversity (measured by the variance of the sum) will be equal to the chance developmental variance (variance of the difference). If other sources of variation are introduced in the studied group of individuals, the level of total phenotypic diversity will be higher than that of chance developmental variability. Thus, by comparing these two variances we can evaluate what fraction of the observed phenotypic diversity is due to stochastic ontogenetic variability (Zakharov 1989).

The value of the difference between the sides of the body is similar to the value of the difference between monozygotic twins (Astauroff 1930, Lundström 1967, Zakharov 1989). In addition, the value 

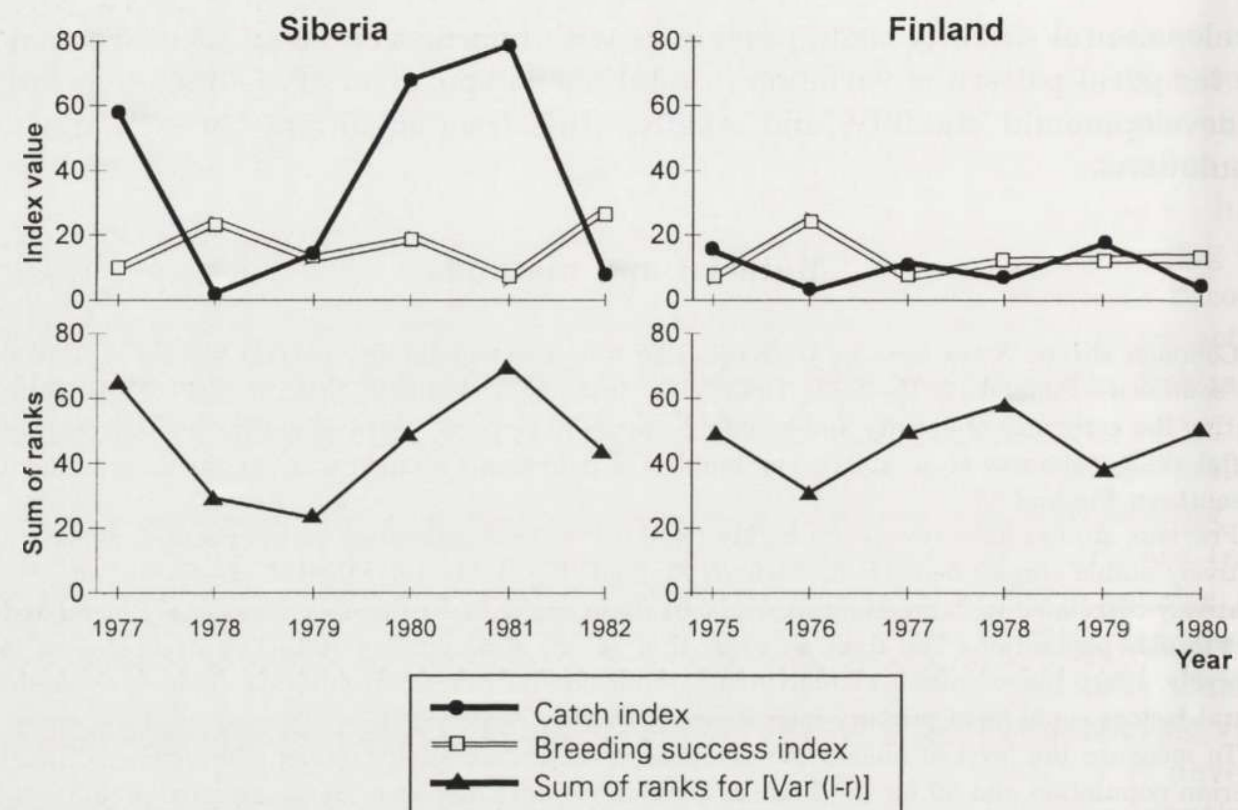

Fig. 1. Changes in population density (catch index - a number of specimens caught by 100 traps per night), breeding success index and chance developmental variance, sum of ranks for [Var (l-r)], of skull foramen numbers in the Siberian and Finnish populations of the common shrew (according to Zakharov et al. 1991).

of the coefficient of correlation between the common measures of heritability for the quantitative characters and measures of difference between the two sides of the body is equal to -1 (Soule 1982, Leary et al. 1985).

To estimate what fraction of the total phenotypic variance calculated for $(l+r)$ was due to variance for $(1-r)$, the two variances were compared by calculating the difference and the ratio between their values for the separate characters. In addition, the geometrical mean of the variances for the entire set of analyzed characters was calculated.

Homogeneity of variances was tested using Bartlett's test and Friedman's test (Sokal and Rohlf 1981). In spite of using 13 characters for the Siberian population and 30 characters for the Finnish population, in all figures data for sum of ranks (calculated in the Friedman's tests) for Finland were scaled by multiplying the original values by $0.43(13 / 30)$ to make them comparable with the ones for Siberia.

\section{Results}

\section{Central Siberia}

Analysis of the dynamics of total phenotypic variance, sum of ranks for [Var $(1+r)]$, revealed significant annual changes (Table 1, Fig. 2), which were correlated neither with breeding success nor with density (Table 1). In spite of a higher level 
Table 1. Between-year variation in measures of phenotypic diversity of the skull foramina numbers in the Siberian and Finnish populations of the common shrew. Friedman's test: \# $p<0.1,{ }^{*} p<0.05$, ${ }^{* *} p<0.01,{ }^{* * *} p<0.001, \mathrm{df}=4$ in all coefficients of correlation (six years).

\begin{tabular}{|c|c|c|c|c|c|c|}
\hline \multirow{3}{*}{ Parameter } & \multicolumn{3}{|c|}{ Siberia } & \multicolumn{3}{|c|}{ South Finland } \\
\hline & \multirow{2}{*}{$\begin{array}{c}\text { Variation } \\
\text { between } \\
\text { years }\end{array}$} & \multicolumn{2}{|c|}{ Correlation with: } & \multirow{2}{*}{$\begin{array}{c}\text { Variation } \\
\text { between } \\
\text { years }\end{array}$} & \multicolumn{2}{|c|}{ Correlation with: } \\
\hline & & density & $\begin{array}{l}\text { breeding } \\
\text { success }\end{array}$ & & density & $\begin{array}{c}\text { breeding } \\
\text { success }\end{array}$ \\
\hline $\begin{array}{l}\text { Sum of ranks for } \\
\text { [Var }(1+r)]\end{array}$ & $19.32^{* * *}$ & +0.19 & +0.40 & $18.49^{* *}$ & -0.29 & -0.46 \\
\hline $\begin{array}{l}\text { Geometrical mean for } \\
{[\operatorname{Var}(1+\mathrm{r})-\operatorname{Var}(1-\mathrm{r})]}\end{array}$ & $10.17 \#$ & -0.35 & +0.63 & 8.80 & -0.68 & +0.17 \\
\hline $\begin{array}{l}\text { Sum of ranks for } \\
{[\operatorname{Var}(1+r)-\operatorname{Var}(1-r)]}\end{array}$ & $24.20 * * *$ & -0.37 & +0.69 & 5.79 & -0.40 & -0.15 \\
\hline $\begin{array}{l}\text { Sum of ranks for } \\
{[\operatorname{Var}(1-r) / \operatorname{Var}(1+r)]}\end{array}$ & $27.67 * * *$ & $+0.78 \#$ & $-0.84^{*}$ & 4.99 & +0.14 & -0.67 \\
\hline
\end{tabular}

of total variance in the last three years than in the first three years, both peak-years (1977 and 1981) usually had lower total phenotypic diversity than the previous years and especially the following years of the minimal density (Fig. 2).

An integrated comparison of changes in total phenotypic variance, geometrical mean for [Var $(\mathrm{l}+\mathrm{r})]$, and chance developmental variance, geometrical mean for [Var (l-r)], for all characters in the Siberian population revealed a clear tendency for more similar values to occur in the peak-years $(1977,1981)$ than in the other years (Fig. 3). The geometrical mean for the difference between these two variances, [Var $(1+r)]$ and [Var $(1-r)]$, showed nearly significant annual changes with positive values of the coefficient of correlation with breeding success and negative with density (Table 1 ).
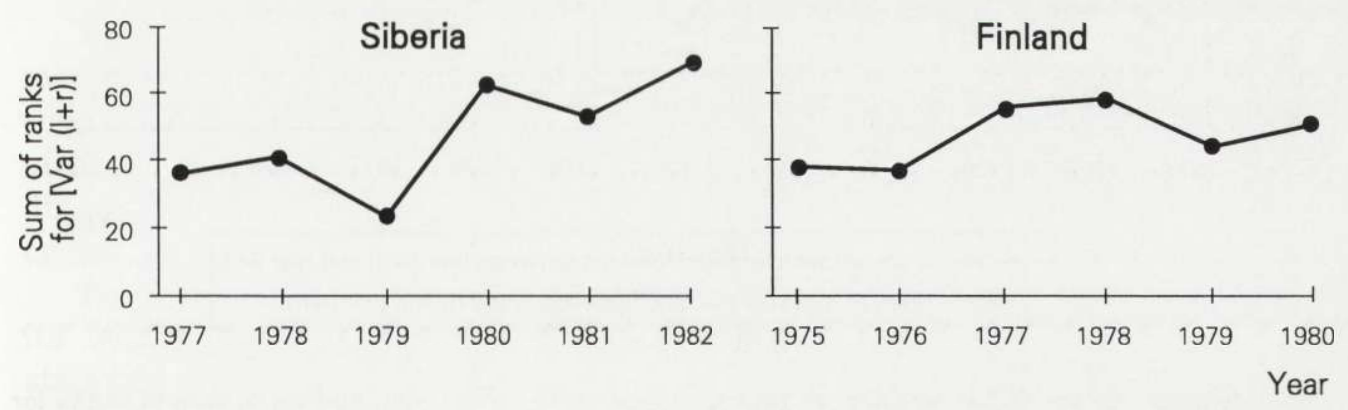

Fig. 2. Changes in the total phenotypic variance, sum of ranks for [Var $(1+r)]$, in two populations of the common shrew. 


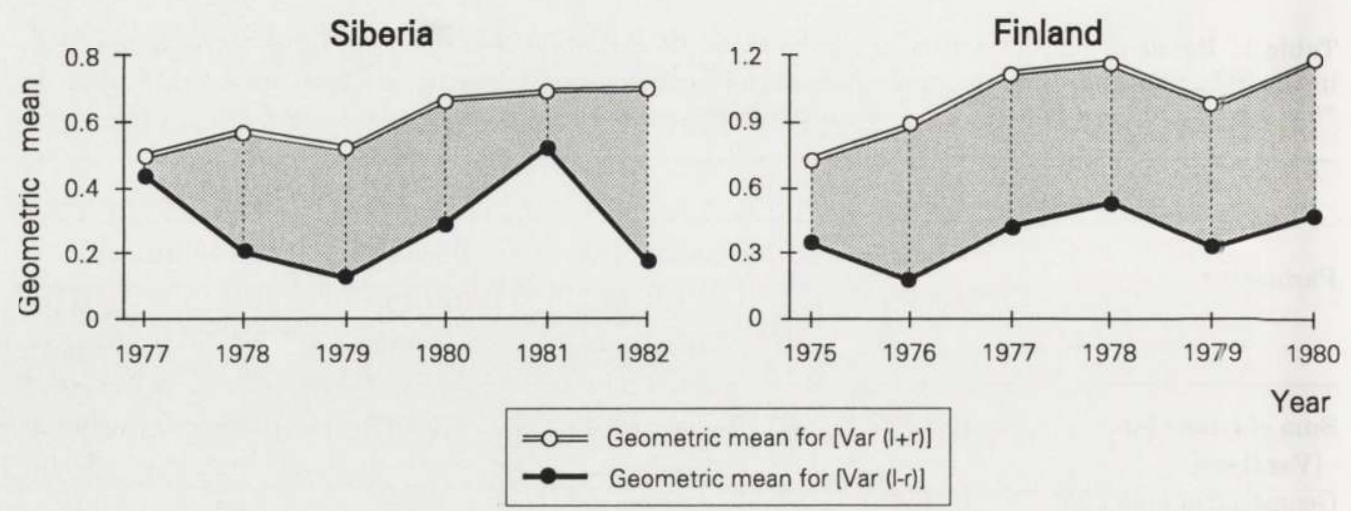

Fig. 3. Comparison of the annual values of the total phenotypic variance, geometrical mean for [Var $(1+r)$, and chance developmental variance, geometrical mean for [Var (l-r)], in two populations of the common shrew.

A non-parametric analysis for the separate characters (Friedman's test) revealed significant changes in the value of the difference between the two variances, sum of ranks for [Var (l+r) - Var (l-r)], (Table 1). Both peak-years (1977 and 1981) had minimal values of such a parameter for the entire study period (Fig. 4). The difference between two variances, sum of ranks for [Var $(l+r)-\operatorname{Var}(1-r)]$, showed positive value of coefficient of correlation with breeding success index and negative with density (Table 1 ).

Another index, a ratio between the values of variance for difference and variance for sum, sum of ranks for [Var $(1-r) / \operatorname{Var}(1+r)]$, also revealed differences between years (Fig. 4). The sum of ranks for this parameter was highest for both peak years $(1977,1981)$ and its annual changes showed positive (nearly significant,
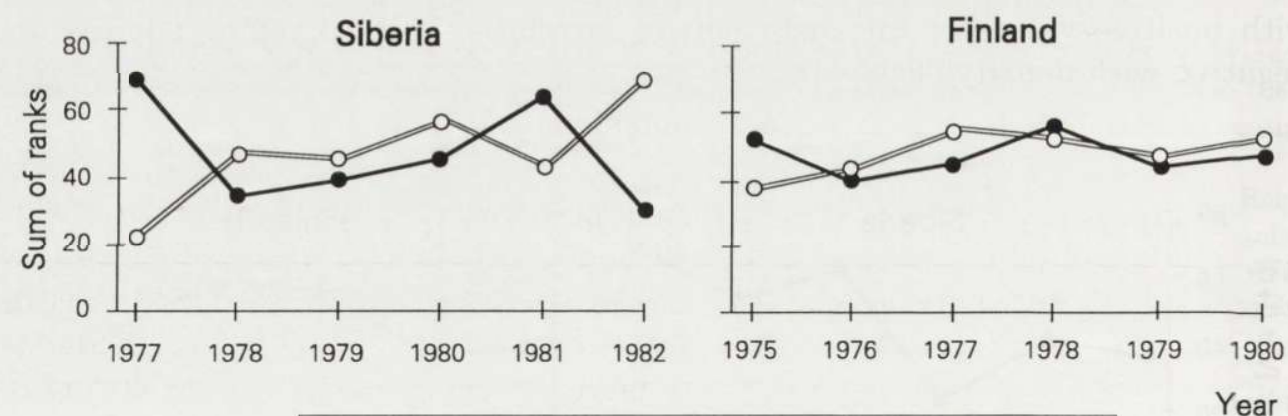

$=0=$ Sum of ranks for the difference between $[\operatorname{Var}(1+r)]$ and $[\operatorname{Var}(1-r)]$

$\rightarrow$ Sum of ranks for the ratio between $[\operatorname{Var}((-r)]$ and $[\operatorname{Var}(1+r)]$

Fig. 4. Changes in the difference, sum of ranks for [Var (1+r) - Var (l-r)], and ratio, sum of ranks for [Var $(1-r) / \operatorname{Var}(l+r)]$, between the total phenotypic variance, $[\operatorname{Var}(l+r)]$, and chance developmental variance, [Var (l-r)], in two populations of the common shrew. 
$p<0.1$ ) value of coefficient of correlation with density and negative (significant, $p<0.05$ ) for breeding success index (Table 1). An essentially similar pattern for the temporal dynamics was found for chance developmental variance as a measure of developmental stability (Fig. 1; Zakharov et al. 1991).

Thus, all the data obtained for the Siberian population revealed that the total phenotypic variance of morphological characters under study showed some annual changes, but they were not correlated with general biological parameters (neither density nor breeding success). An attempt to compare this pattern with the annual changes in chance developmental variance did not reveal any correlation between them. All estimates of the role of such developmental variation in total phenotypic diversity revealed an increase in its proportion in years of highest population density.

\section{Southern Finland}

The total phenotypic variance, sum of ranks for [Var $(1+r)]$, in the Finnish shrew population showed significant year to year changes with non-significant negative values of the coefficient of correlation with breeding success and density (Table 1, Fig. 2). None of the estimates of the variance for difference as a proportion of the variance for the sum [neither differences, geometrical mean for [Var $(l+r)$ - Var (l-r)], sum of ranks for [Var $(1+r)$ - Var (l-r)], nor the ratio between them, sum of ranks for [Var $(l+r) / \operatorname{Var}(1-r)]\}$ exhibited any significant variation between years (Table 1, Figs 3 and 4 ).

Thus, the Finnish population exhibited significant changes in the level of phenotypic diversity that were not correlated with general biological parameters. These changes proved to be similar with the pattern of dynamics of the chance developmental variance (Figs 1 and $2 ; r=0.78, p<0.1$ ). The ratio between the total variance and the chance developmental variance (measured by different estimates) did not significantly vary during the study period.

\section{Discussion}

Significant temporal changes in phenotypic diversity for the characters of skull morphology were detected for both cyclic and noncyclic populations of shrews, but they were not clearly correlated with either density or breeding success in either of them. These results in general, and particularly the fact that total phenotypic diversity did not increase when density increased more than 70 times during the cycles in the Siberian population (Sheftel 1989), seems unexpected.

To understand the nature of the observed pattern of phenotypic diversity and its temporal changes, we compared its value with measures of developmental stability. We did this by comparing the variance for the sum (as a measure of the total phenotypic diversity) with the variance for the difference (as a measure of a chance developmental variability) in values of characters on two sides of the skull. 
These two measures of variation proved not to be correlated with each other in the cyclic Siberian population. Earlier we found (Zakharov et al. 1991), that the level of developmental stability was the lowest (corresponding to the highest values of chance developmental variance) during the peak year of the population cycles, but this was not also true for the total phenotypic variance (Figs 1 and 2).

An increase in the chance developmental variance in the years of maximal density was not accompanied by an increase of the total phenotypic variance. This means that the proportion of the chance variance of the total variance becomes higher in the years with the higher density, while the proportion of other kinds of variation decreases. We assume that it is primarily actual for the genetic variation, because the variety of environments in the certain area under study inhabited by the same population commonly proves to be rather stable in time. Two measures of such changes (difference and ratio between the two variances compared, including parametric and nonparametric tests) support such a conclusion (Figs 3 and 4). At first sight such a result seems to be unexpected and less probable. But keeping in mind what processes can take place in a population at different phases of the cycle it could be considered as rather natural.

During the years of lower population density, following the years of maximal density, the total number of individuals inhabiting the same territory was very small. In such a situation, natural selection should be rather weak. This situation may produce high genotypic diversity in a relatively small population and low phenotypic diversity derived from the accidents during individual development.

During the years of high density, at least in populations where overpopulation has a serious stress effect (such as the Siberian population in the present study), just the opposite situation should occur. A sharply increased number of individuals could lead to an increase in genotypic diversity, but these individuals are survivors after serious selection pressure. It has already been established for this population (Sheftel 1989), just for these particular years, that the rate of prenatal and postnatal mortality is the highest in comparison with all other years. In such a case, most extreme phenotypes (corresponding to extreme genotypes) are commonly eliminated from the population. This means that the increased number of individuals should be accompanied by a decreased level of phenotypic diversity determined by differences in genotypes. At the same time, individuals that do not die have decreased developmental stability as a result of stress (Zakharov et al. 1991). Such a decrease can be easily detected by small phenotypic deviations from the norm, as a manifestation of developmental noise (detected in this study by chance developmental variance). These small deviations most probably are not so important for the viability of individuals and can be allowed by natural selection.

The population from southern Finland illustrates another and probably more common type of temporal change in phenotypic diversity. While changes in phenotypic diversity here are not correlated with density, there is an indication of a negative correlation with breeding success, that was established earlier for developmental stability measures (Zakharov et al. 1991). Contrary to the Siberian 
population, here is an indication for a positive correlation between the total phenotypic variance and chance developmental variance. This means that the changes in phenotypic diversity in this population mainly reflect changes in developmental stability, while the proportion of all other components of total phenotypic variance is relatively stable.

A possible explanation is that density oscillations in the Finnish shrew population are not strong enough to affect genotypic diversity. Moreover, they also have no effect on developmental stability which is not correlated with density (Zakharov et al. 1991). Small alterations in the condition of developing individuals (depending perhaps on environmental factors, Pankakoski 1985), resulting in changes of developmental stability, actually determines oscillations in the level of the total phenotypic diversity.

\section{Conclusions}

In general, our results illustrate that not only dynamics of genotypic variety (as is commonly assumed), but also the alterations in the level of developmental stability can be of great importance for changes in phenotypic diversity. They also demonstrate that the comparison of the measures of phenotypic diversity and developmental stability provides us with an opportunity to understand more about the processes taking place in populations having different types of dynamics.

Acknowledgements: We are very grateful to Prof R. Palmer and three anonymous referees provided very helpful comments and corrections of English. Grant from the Academy of Finland, and from Maj and Tor Nessling Foundation are gratefully acknowledged.

\section{References}

Astauroff B. L. 1930. Analyse der erblichen Storungsfalle der bilateralen Symmetrie im Zusammenhang mit der selbstständigen Variabilität änlicher Strukturen. Zeitschrift für inductive Abstammungs- und Vererbungslehre 55: 183-262.

Bader R. S. 1965. Fluctuating asymmetry in the dentition of the house mouse. Growth 29: 291-300. Langlet O. 1971. Revising some terms of intraspecific differentiation. Hereditas 68: 277-280.

Leary R. F. and Allendorf F. W. 1989. Fluctuating asymmetry as an indicator of stress: implications for conservation biology. Trends in Ecology and Evolution 4: 214-217.

Leary R. F., Allendorf F. W. and Knudsen K. L. 1985. Inheritance of meristic variation and the evolution of developmental stability in rainbow trout. Evolution 39: 308-314.

Leary R. F., Allendorf F. W. and Knudsen K. L. 1992. Genetic, environmental, and developmental causes of meristic variation in rainbow trout. Acta Zoologica Fennica 191: 79-95.

Lundström A. 1967. Genetic aspects of variation in tooth width based on asymmetry and twin studies. Hereditas 57: 401-410.

Mather K. 1953. Genetical control of stability in development. Heredity 7: 297-336.

Palmer A. R. and Strobeck C. 1986. Fluctuating asymmetry: measurement, analysis, patterns. Annual Review Ecology and Systematics 17: 391-421.

Pankakoski E. 1985. Relationship between some meteorological factors and population dynamics of Sorex araneus in southern Finland. Acta Zoologica Fennica 173: 287-289. 
Pankakoski E. and Hanski I. 1989. Metrical and non-metrical skull traits of the common shrew Sorex araneus and their use in population studies. Annales Zoologici Fennici 26: 433-444.

Pankakoski E., Koivisto I. and Hyvärinen H. 1992. Reduced developmental stability as an indicator of heavy metal pollution in the common shrew Sorex araneus. Acta Zoologica Fennica 191: 137-144.

Pankakoski E., Väisänen R. A. and Nurmi K. 1987. Variability of muskrat skulls: measurement error, environmental modification and size allometry. Systematic Zoology 36: 35-51.

Philiptschenko Yu. 1978. [Variability and the methods of its study.] Nauka, Moskva: 1-239. [In Russian]

Rasmuson M. 1960. Frequency of morphological deviations as a criterion of developmental stability. Hereditas 46: 511-536.

Reeve E. C. R. 1960. Some genetic tests on asymmetry of sternopleural chaeta number in Drosophila. Genetic Research 1: 151-172.

Sheftel B. I. 1989. Long-term and seasonal dynamics of shrews in Central Siberia. Annales Zoologici Fennici 26: 357-370.

Siegel M. I., Mooney M. P. and Taylor A. B. 1992. Dental and skeletal reduction as a consequence of environmental stress. Acta Zoologica Fennica 191: 145-149.

Sokal R. R. and Rohlf F. J. 1981. Biometry. Freeman, San Fransisco: 1-859.

Soulé M.1967. Phenetics of natural populations. II. Asymmetry and evolution in a lizard. American Naturalist 101: 141-160.

Soulé M. 1982. Allomeric variation. I. The theory and some consequences. American Naturalist 120: 751-764.

Thoday J. M. 1953. Components of fitness. Symposium of Society of Experimental Biology 7: 96-113.

Thoday J. M. 1958. Homeostasis in a selection experiment. Heredity 12: 401-415.

Valentine D. W., Soulé M. E. and Samollow P. 1973. Asymmetry analysis in fishes: a possible statistical indicator of environmental stress. Fishery Bulletin 71: 357-370.

Van Valen L. 1962. A study of fluctuating asymmetry. Evolution 16: 125-142.

Yablokov A. V. 1986. Population biology. Progress and problems of studies on natural populations. Advances in Science and Technology in the USSR, Biology Series. Mir Publishers, Moscow: 1-303.

Zakharov V. M. 1981. Fluctuating asymmetry as an index of developmental homeostasis. Genetica (Beograd) 13: 241-256.

Zakharov V. M. 1987. [Animal asymmetry: Population-phenogenetic approach.] Nauka, Moskva: 3-213. [In Russian]

Zakharov V. M. 1989. Future prospects for population phenogenetics. Soviet Scientific Reviews, Sec. F, Physiology and General Biology Reviews 4 (3): 1-79.

Zakharov V. M. 1992. Population phenogenetics: Analysis of developmental stability in natural populations. Acta Zoologica Fennica 191: 7-30.

Zakharov V. M., Pankakoski E., Sheftel B. I., Peltonen A. and Hanski I. 1991. Developmental stability and population dynamics in the common shrew, Sorex araneus. American Naturalist 138: $797-810$.

Received 7 December 1995, revised 28 May 1997, accepted 12 June 1997. 